\title{
IGS Combined and Contributed Earth Rotation Parameter Solutions
}

\author{
Jan Kouba \\ Geodetic Survey of Canada, 615 Booth Street, Ottawa, Ontario K1A \\ oE9, Canada (e-mail kouba@geod.emr.ca) \\ Gerhard Beutler and Markus Rothacher \\ Astronomical Institute, University of Bern, Sidlerstrasse 5, CH-3012 \\ Bern,Switzerland (e-mail beutler@aiub.unibe.ch)
}

\begin{abstract}
Since January 1995 the International GPS Service (IGS) has been combining and analyzing daily polar motion (PM) series, produced and submitted by seven IGS analysis centers (ACs) for the IGS Final orbit/clock combinations. Since June 30, 1996 the IGS Earth Rotation Parameter (ERP) series that accompany the IGS combined orbits, also include combined PM rates. Furthermore, since March 1997, the IGS LOD (Length of Day) solutions are based on separate combinations of AC LOD solutions calibrated and weighted according to the IERS Bulletin A definite values. Similar to $\mathrm{AC}$ orbit solutions, the PM solutions have improved considerably since 1995 , so that currently the IGS combined and the best $\mathrm{AC}$ PM solutions are at or below the 0.1 mas precision level, although PM biases may exceed .1 mas. Comparisons of AC ERP and PM rate solutions with the IGS Final combined ERP series revealed signals with 7 and 14-day periods for some AC solutions.

During 1998, the IGS Final and the best AC PM rate solutions compared with Atmospheric Angular Momentum (AAM) at 0.3 mas/day (rms) with an average correlation of about 0.8 and 0.6 for the PM $x$ and $\mathrm{PM}$ y rate components. The correlation varied considerably with time and frequency, though significant correlation already started from 6-day periods and reaching maxima within 10 to 50 day period bands. Most of the remaining signal in the PM rate solutions could likely be accounted for by Ocean Angular Momentum (OAM) as seen from the comparisons of combined OAM and AAM with the IGS PM series during 1995 and early 1996 when also OAM data were made available. During this period the IGS PM rates agreed with the combined OAM + AAM series with 0.3 and 0.2 mas/day (rms) for the PM x and y components and with an average correlation of about 0.8 for both PM components.
\end{abstract}

\section{Introduction}

The high correlation between $\mathrm{PM}$ and the $\mathrm{X}, \mathrm{Y}$ - rotations of the corresponding orbit solutions was recognized from the inception of IGS orbit combinations 
(Springer and Beutler, 1993). That is why the AC PM solutions were initially used to orient the $\mathrm{AC}$ orbit solutions to a reference $\mathrm{PM}$ series prior to the IGS orbit combinations. From January 1, 1994, the IERS Bulletin A and B series were adopted as the reference PM series for the official IGS Rapid and Final orbit combinations. So that the IGS Rapid (IGR) during 1994 through the first part of 1995 and 1994 till June 30, 1996 for the IGS Final orbit combinations, the corresponding PM and LOD (ERP) series were based on the IERS Bulletin $A$ and Bulletin B, respectively. The two distinct IGS combinations, the Rapid and Final are respectively based on the $\mathrm{AC}$ rapid and final solutions, and they differ in submission delays, amounts of data processed and for some ACs, also in the processing strategies used. The IGS Rapid combinations (IGR) include an additional AC, USNO (US Naval Observatory), which has elected to participate only in the IGR combinations.

The initial experience and the steady improvements of all $\mathrm{AC}$ solutions during 1994, the first year of IGS combination, indicated that AC PM solutions were quite consistent and the prior PM orbit alignment was no longer needed. Subsequently, separate orbit and PM combinations were introduced, both without any prior alignment to a reference PM series, though in order to maintain the orbit/PM consistency, the orbit weights from the orbit combination process (Beutler et al., 1995) were also retained for the PM combinations. The adopted set of the 13 reference station positions and velocities (Kouba and Mireault, 1996) provided the ITRF (International Terrestrial Reference Frame) realization for both the IGS orbit and PM combined series, namely ITRF93 from January 1, 1995 to June 29, 1996 and ITRF94 for June 30, 1996 till February 28, 1998. For completeness so that an IGS combined PM series is available starting from January 1, 1995, the IGR orbit and PM combinations (in ITRF93) were recomputed back to January $1,1995$.

On June 30, 1996, apart from the change to a more precise ITRF94 realization, a number of significant changes and improvements were introduced by all $\mathrm{ACs}$, namely the use of subdaily ERP model became mandatory and all ACs also started to include separate PM rate solutions. Furthermore, the AC submission and IGS combination deadlines were considerably shortened from weeks to months, down to less than $24 \mathrm{~h}$ for IGR and within 10 days for the IGS Final solutions. Since March 1, 1998, IGS has used ITRF96, which itself is nominally the same as ITRF94. The ITRF96 is realized with up to 47 ITRF96 station position and velocities (Boucher et al., 1998). From March 1997, the IGS combined ERP also include separated LOD combinations, calibrated according to the IERS Bulletin A as well as the UT values obtained by integrating the combined LOD and aligned according to definitive Bulletin A UT1 values (Kouba and Mireault, 1997; Mireault et al., 1999).

\section{1996-1998 IGS and AC ERP series}

Since June 30, 1996 the IGS combined and AC PM series are relatively consistent and refer, at least nominally, to the same reference ITRF94 reference frame. Thus the period of June 30, 1996 to December 1998 was selected for the detailed comparisons and analyses of the final AC PM and PM rate solutions. The comparison statistics of the individual final AC PM solutions with respect to the 
IGS PM combinations are summarized in Table 1 ; here the average $\mathrm{AC}$ biases are within 0.1 or .2 mas for most ACs. The best AC PM solutions compare to IGS Final PM with an rms of about 0.1 mas during this period. For completeness the UT1 equivalent (UT) values included in the AC ERP submissions are also shown here. Since satellite solutions are insensitive to UT changes, there are considerable differences amongst ACs. Some ACs (e.g. NGS, SIO) simply use the best available IERS (Bulletin A) values, while some ACs integrate their LOD solutions with occasional resets (e.g. COD), yet others have maintained continuous UT solutions for considerable times without any resets (e.g. EMR, JPL).

In order to establish the precision and stability of the IGS ERP series the IERS ERP series, the Bulletin A and C04 (the continuous version of the Bulletin B) ERP series were also compared to the IGS Final ERP in Table 1 and Figure 1. As one can see both IERS PM series agree quite well with IGS at about the 0.1 mas level, though PM biases can exceed these levels. Even differences between the two IERS ERP series also can exceed the .1 mas level. Up to March 2, 1997 (MJD 50509) the IGS ERP series contained only the latest Bulletin A UT1 values that were available at the time of combinations, usually one to three day predictions of UT1. On March 2, 1997 a separate IGS LOD combination was initiated and the IGS UT values are based on the combined LOD, integrated typically for 1-3 days from the last stable Bulletin A UT1 value. Figure 1 (bottom) clearly shows the improved performance of the IGS combined UT after MJD 50509.

Table 1. ERP differences with respect to the IGS Final ERP (ITRF94, ITRF96; interval: June 30, 1996-December 31, 1998)

\begin{tabular}{lcccccc} 
AC/ERP & \multicolumn{2}{c}{$\mathrm{PMx}(\mathrm{mas})$} & \multicolumn{2}{c}{$\mathrm{PMy}(\mathrm{mas})$} & \multicolumn{2}{c}{$\mathrm{UT}(\mathrm{ms})$} \\
& Mean & rms & Mean & rms & Mean & rms \\
\hline COD & 0.055 & 0.132 & -0.208 & 0.197 & -0.238 & 0.308 \\
EMR & -0.127 & 0.194 & 0.277 & 0.213 & -11.023 & 2.279 \\
ESA & -0.062 & 0.203 & 0.128 & 0.245 & 0.023 & 0.193 \\
GFZ & 0.040 & 0.112 & 0.028 & 0.140 & 0.993 & 1.489 \\
IGR & 0.052 & 0.210 & 0.084 & 0.245 & -0.010 & 0.329 \\
JPL & -0.080 & 0.116 & -0.048 & 0.137 & -3.990 & 5.665 \\
NGS & 0.110 & 0.478 & 0.225 & 0.505 & -0.005 & 0.459 \\
SIO & 0.142 & 0.494 & 0.043 & 0.477 & -0.005 & 0.151 \\
& & & & & & \\
BULL-A & -0.295 & 0.067 & -0.113 & 0.099 & -0.007 & 0.041 \\
C 04 & -0.249 & 0.126 & 0.028 & 0.140 & -0.011 & 0.045 \\
\hline
\end{tabular}

The small step of about 0.1 mas in PM y at MJD 50873 in Figure 1 is due to the ITRF94-ITRF96 difference, which is consistent with an estimated PM y shift of about $0.1 \mathrm{mas}$ (Kouba, 1999). However, the small PM y discontinuity at about MJD 50480 was likely caused by modeling changes for some AC solutions and it demonstrates the stability limit of IGS PM series prior March 1, 1998 when the more precise realization of ITRF96 has been adopted by IGS. The 
improved IGS PM stability and rms after March 1 (MJD 50873) is also shown in Fig.1. This is even better seen in Table 2, which contains means and rms of the AC PM and UT comparisons, but only for the ITRF96 period of March 1December 31, 1998. During this period, the IGS Rapid (IGR) combination and the best AC and both IERS PM series agreed well below the 0.1 mas level with the IGS Final PM. IGR shows perhaps the most significant improvements, when Table 2 and Table 1 are compared. The improved UT comparisons shown here for the Bull. A and $\mathrm{C} 04$ series is mainly due to improvements of $\mathrm{AC}$ modeling and/or the IGS LOD / UT combinations, rather than the effect of the new ITRF96 realization.

Table 2. ERP Differences with respect to IGS Final ERP (ITRF96; March 1 - December 31, 1998)

\begin{tabular}{lcccccc} 
AC/ERP & \multicolumn{2}{c}{ PMx(mas) } & \multicolumn{2}{c}{ PMy(mas) } & \multicolumn{2}{c}{$\mathrm{UT}(\mathrm{ms})$} \\
& Mean & rms & Mean & rms & Mean & rms \\
\hline COD & 0.048 & 0.088 & -0.038 & 0.093 & -0.166 & 0.257 \\
EMR & -0.158 & 0.197 & 0.176 & 0.190 & -13.294 & 1.618 \\
ESA & 0.007 & 0.156 & 0.706 & 0.223 & 0.038 & 0.090 \\
GFZ & 0.013 & 0.073 & -0.021 & 0.086 & 0.014 & 0.081 \\
IGR & -0.009 & 0.096 & 0.061 & 0.101 & 0.010 & 0.079 \\
JPL & -0.032 & 0.071 & -0.034 & 0.091 & -10.857 & 1.887 \\
NGS & -0.227 & 0.404 & 0.180 & 0.478 & 0.002 & 0.377 \\
SIO & 0.001 & 0.117 & 0.061 & 0.134 & -0.015 & 0.098 \\
& & & & & & \\
BULL-A & -0.299 & 0.049 & -0.039 & 0.078 & -0.007 & 0.023 \\
C 04 & -0.220 & 0.062 & -0.022 & 0.058 & -0.005 & 0.032 \\
\hline
\end{tabular}

Figures 2 and 3 show the spectra of PM x, y differences with respect to the IGS Final PM for IGR, IERS and the seven AC PM solutions during 1998. Note the progressively larger scale from the top to the bottom groups. The two top plots show periods (in days) and amplitudes (in .01 mas) of the IGR and AC PM differences with respect to IGS. For comparison purposes the bottom parts of the figures contain the spectra of the differences amongst the IGS, C04 and Bulletin A PM series.

As seen from Figure 2, the spectra of IGR and AC PM x differences are relatively well behaved with the exception of two $\mathrm{AC}$ solutions that show some peaks at the 7, 14 and 73 day periods. The corresponding spectra for the C04IGS and C04-Bull. A differences are also quite well behaved, with only two large peaks, both for the C04-IGS PM x difference. The first, at the 3.7 day period, which even if real, could hardly be seen for C04-Bull. A due to C04/Bull. A smoothing at or below the 2 day periods. This smoothing is clearly visible in Figures 2 and 3, i.e., the amplitudes of the C04-Bull. A PM amplitudes near the 2 day period are zero. The second large peak at the period of 51 days does not appear in the AC PM x spectra and it is only partly shown for the C04-Bull. A differences. It may be due to IGS and it may simply reflect different treatments (e.g. weighting) of the IGS PM contributions in both IERS combined series. 


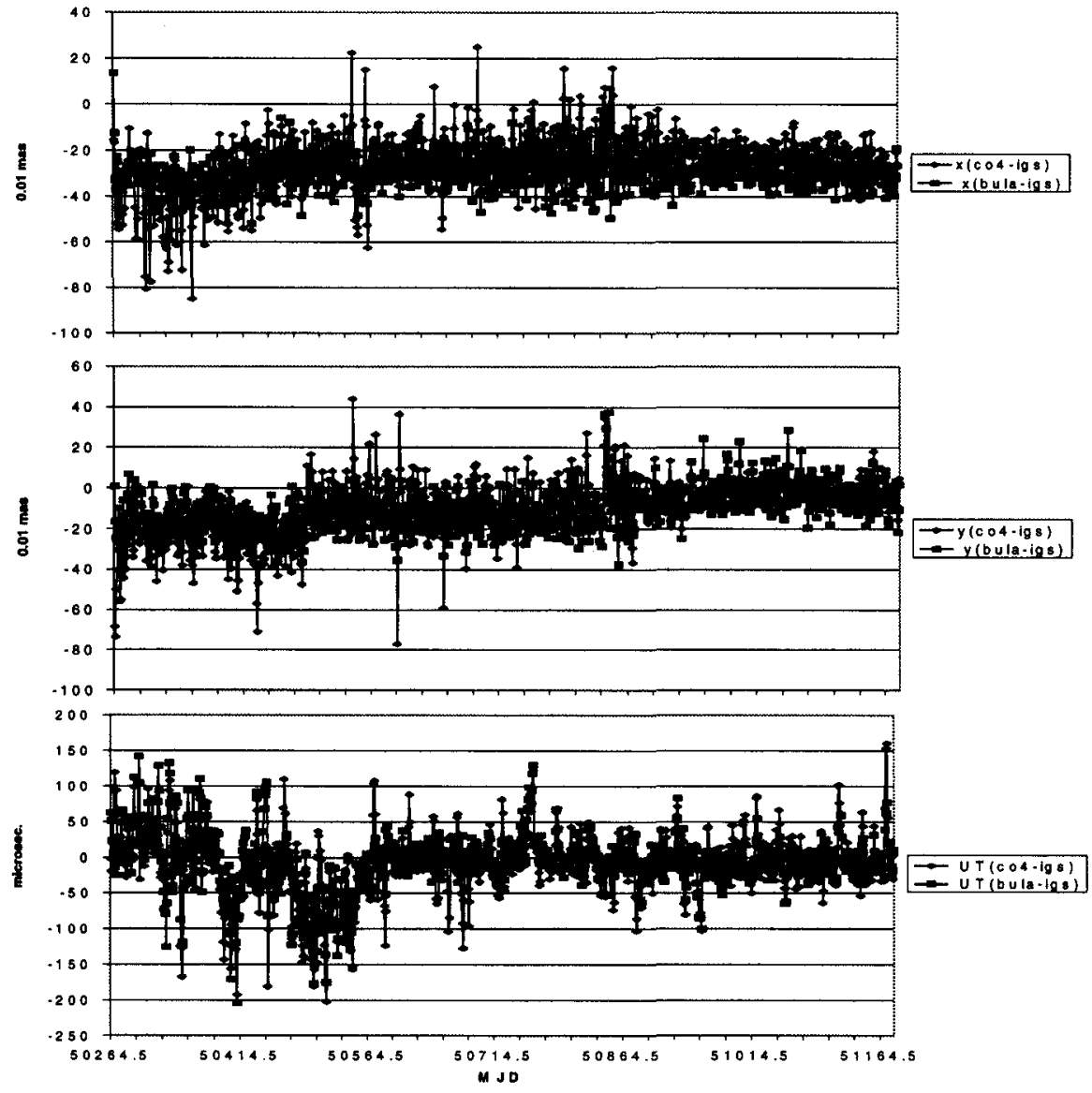

Figure 1. IERS C04 and Bulletin A ERP differences with respect to IGS Final combined ERP during June 30 1996-December 31, 1998. 

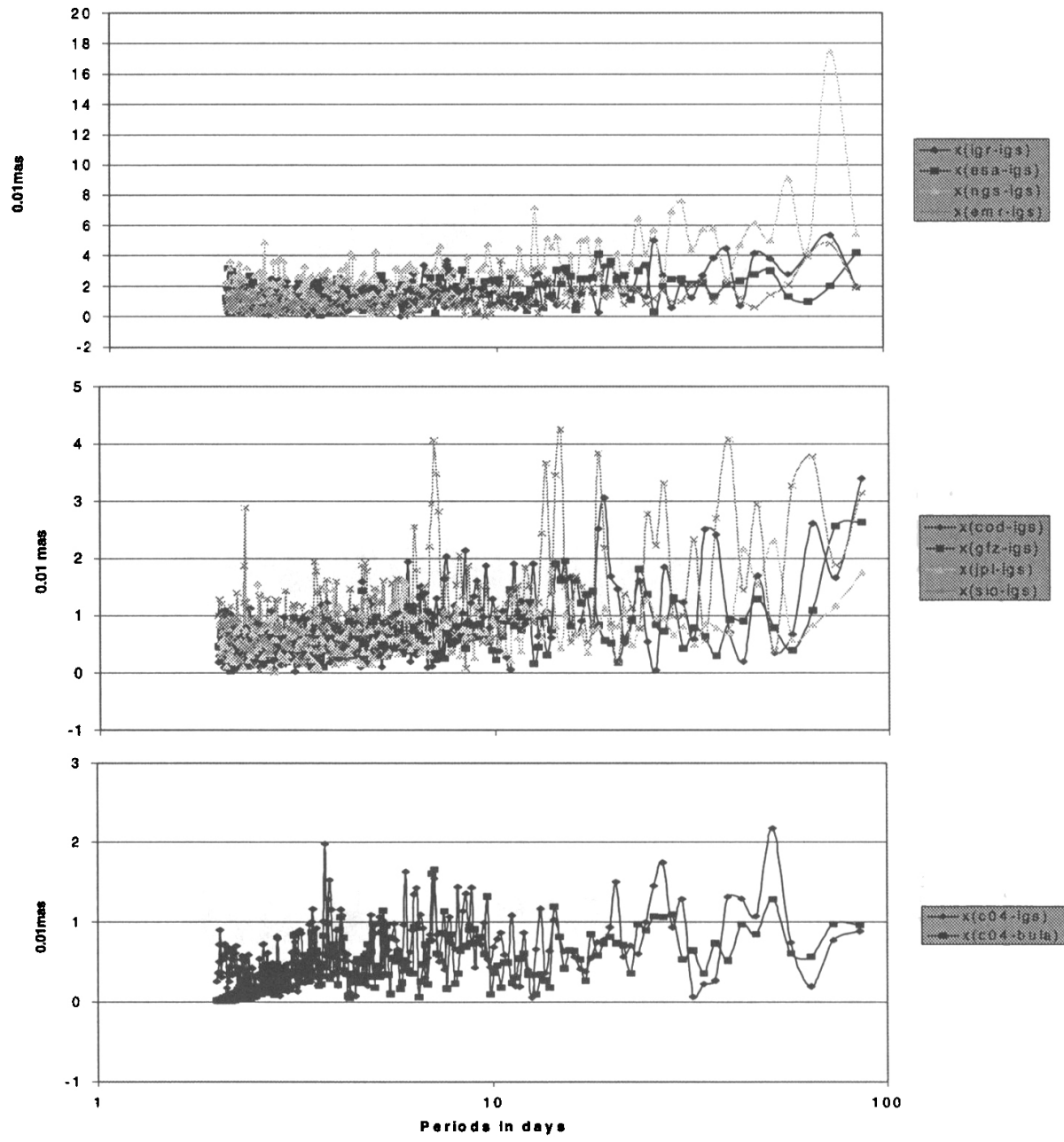

Figure 2. Polar Motion (PM) x amplitudes and periods with respect to IGS Final PM. 
The spectra of the corresponding PM y differences are considerably more variable, with more significant peaks for more $\mathrm{ACs}$, than for the $\mathrm{PM} \times \mathrm{x}$ components. This is to be expected as PM y IGS solutions are generally weaker and less stable than the corresponding PM x solutions. This is likely due to the geometry of the 13 ITRF stations used for the ITRF94 realization up to February 28, 1998. However, by far, the most pronounced is the 7-day peak for the C04-Bull. A difference shown in the bottom part of Figure 3. This 7-day periodic signal has already been confirmed by USNO and subsequently has been practically eliminated by simply changing, within the Bulletin A PM combination, the treatment of the two, weekly alternating, VLBI contributions (J. Ray, 1999 , private comm.).

\section{1996-1998 AC and IGS ERP Rate Series}

Since June 30, 1996 both IGS Combined ERP series (Rapid and Final) also contain combined PM rate values that are obtained as weighted averages of all submitted AC PM rate solutions without any alignment or calibration, while utilizing orbit weights, a process completely analogous to the IGS PM combinations (see e.g. Kouba and Mireault, 1997). Table 3 shows the statistics (means and rms) of ERP rate (PM rate and LOD) differences with respect to the IGS Final for IGR and all AC final solutions for the period of June 30, 1996 to December 31, 1998. Similar statistics tables for both ERP and ERP rates are included every week in the IGS Final combination summary reports (http: ://igscb.jpl.nasa.gov//igscb/mail/igsreports/).

There is considerable variation in $\mathrm{AC}$ approaches and $\mathrm{AC}$ ERP rate solution performance. The best AC PM rate solutions, which also enforce a complete or partial continuity conditions for PM and PM rates and may also employ intervals longer than $24 \mathrm{~h}$, usually show the best performance at about $0.1 \mathrm{mas} / \mathrm{d} \mathrm{rms}$ level, with practically zero mean biases. Similarly for LOD, the solutions with averaging intervals longer than $24 \mathrm{~h}$ usually show the best LOD performance. Tests of PM and PM rate continuity and consistency are shown in the last four columns of Table 3, labeled as "PM/PMrt Consist.". Here the columns lists the means and rms of differences between two PM values at $0 \mathrm{~h}$ UT, interpolated from the subsequent and preceding daily PM (at $12 \mathrm{~h}$ UT epochs) while using the respective PM rate values. The ACs that employ full or partial continuity constraints can be clearly identified by having nearly zero means and rather small rms (see the last four columns of Table 3). If no correlation between PM and PM Rate solution is assumed, then the resulting continuity difference will have the following variance:

$$
\sigma_{C T}^{2}=2 \sigma_{P M}^{2}+\frac{1}{2} \sigma_{R t}^{2},
$$

where $\sigma_{P M}$ and $\sigma_{R t}$ are AC PM and PM rate sigmas, respectively. Furthermore here the continuity mean values are equal to the average bias of the $\mathrm{AC} P M$ rate solutions with respect to the corresponding AC PM solutions. Note that the PM rate means and rms of the first four columns correspond quite well to the continuity means and rms listed in the last four columns. This indicates that the IGS PM rates are nearly unbiased (see the continuity means in the last line 

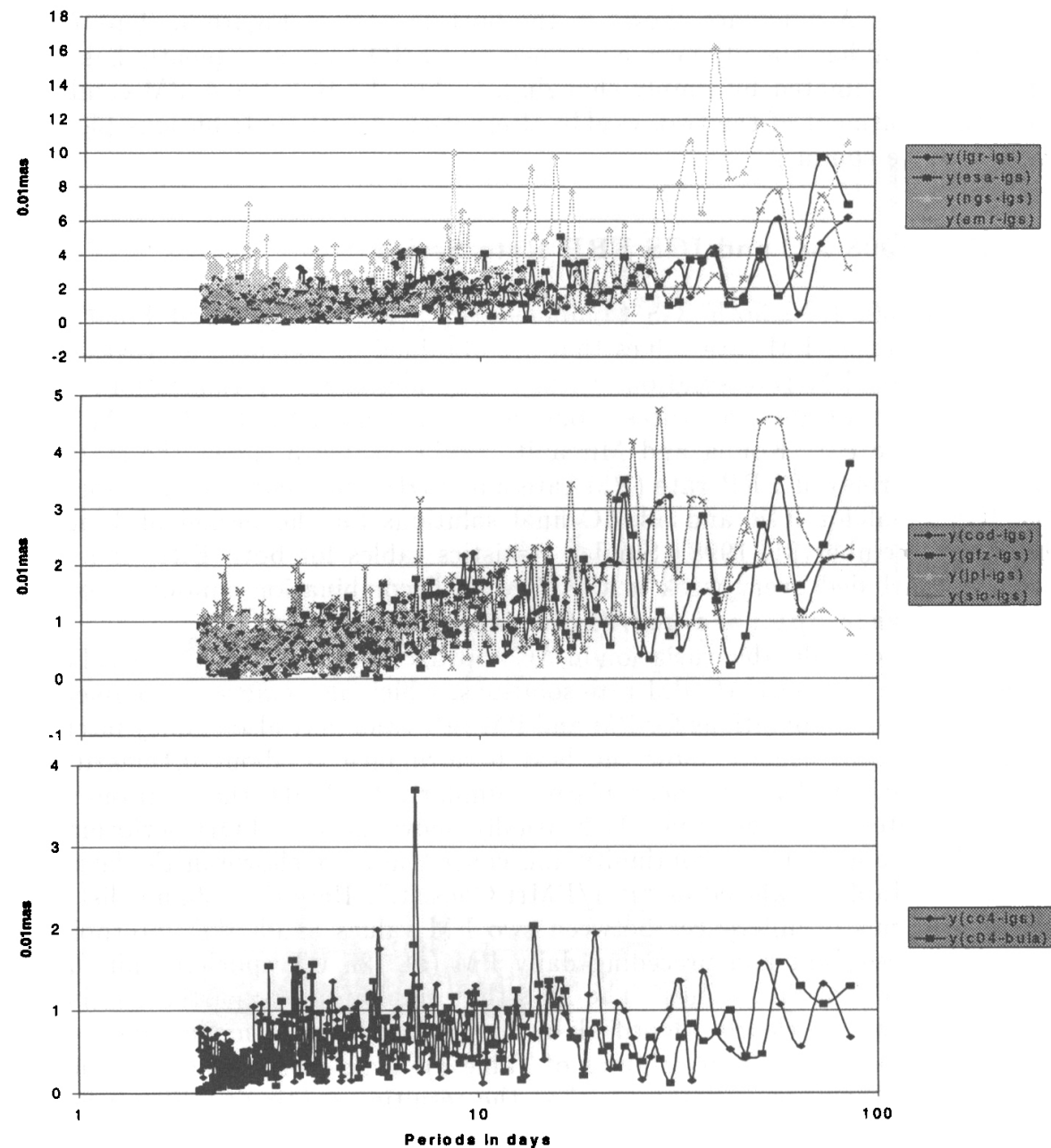

Figure 3. Polar Motion (PM) y amplitudes and periods with respect to IGS Final PM. 
of Table 3) as well as that for the most ACs the adjacent PM rate solutions are highly correlated (correlation $\sim 1$ ).

Table 3. AC PM rate differences with respect to IGS Final PM rate and $\mathrm{AC} P M / P M$ rate consistency (PM differences at $0 \mathrm{~h}$ UT interpolated from subsequent and preceding $\mathrm{PM} / \mathrm{PM}$ rate values at $12 \mathrm{UT}$ ), from June 30, 1996 to December 31, 1998.

\begin{tabular}{lcccccccccc} 
& \multicolumn{9}{c}{ AC-IGS (mas/d) } & \multicolumn{4}{c}{ PM/PMrt Consist. } \\
AC & \multicolumn{2}{c}{ Xrt } & \multicolumn{2}{c}{ Yrt } & \multicolumn{2}{c}{ LOD $(\mu \mathrm{s})$} & \multicolumn{2}{c}{$\Delta \mathrm{X}(\mathrm{mas})$} & \multicolumn{1}{c}{$\Delta \mathrm{Y}$ (mas) } \\
& Mean & rms & Mean & rms & Mean & rms & Mean & rms & Mean & rms \\
\hline COD & 0.055 & 0.132 & 0.029 & 0.139 & 12.8 & 18.4 & -0.007 & 0.061 & -0.005 & 0.069 \\
EMR & -0.485 & 0.535 & -0.051 & 0.537 & 1.0 & 30.9 & -0.549 & 0.509 & -0.081 & 0.521 \\
ESA & 0.147 & 0.395 & -0.006 & 0.461 & -15.1 & 39.9 & 0.087 & 0.347 & -0.042 & 0.404 \\
GFZ & 0.040 & 0.151 & 0.005 & 0.166 & -1.4 & 18.2 & -0.023 & 0.076 & -0.026 & 0.093 \\
IGR & 0.083 & 0.293 & 0.115 & 0.274 & -2.6 & 21.6 & 0.021 & 0.320 & 0.083 & 0.290 \\
JPL & -0.042 & 0.226 & -0.105 & 0.236 & 19.3 & 28.4 & -0.106 & 0.261 & -0.136 & 0.286 \\
NGS & -0.179 & 0.729 & 0.459 & 0.852 & -2.0 & 73.8 & -0.239 & 0.675 & 0.426 & 0.801 \\
SIO & 0.076 & 0.145 & 0.021 & 0.162 & 5.4 & 36.0 & 0.014 & 0.131 & -0.011 & 0.114 \\
IGS & 0.000 & 0.000 & 0.000 & 0.000 & 0.0 & 0.0 & -0.063 & 0.096 & -0.032 & 0.098 \\
\hline
\end{tabular}

\section{ERP rates and atmospheric angular momentum (AAM)}

A strong temporal correlation between LOD and AAM on a time scale of about 50 days has been observed by several authors (e.g. Langley et al., 1981). Barnes et al., (1983) has confirmed the LOD/AAM correlation and has also demonstrated correlation between PM and the equatorial components of AAM at this long period of about 50 days. Eubanks et al. (1988) has studied AAM spectra and predicted a significant PM signal of the AAM origin around the 10-day period. The IGS daily PM series during 1997 confirmed this expectation. In fact, it helped to identify an excessive smoothing of the IERS PM series (Kouba, 1996) which was subsequently corrected for both IERS series. Since June 30, 1996 the IGS combined PM is ideally suited for the AAM/ERP correlation studies, thanks to the highly precise, continuous and unsmoothed daily PM and PM rate solutions. Following the formulation introduced in Barnes et al. (1983) which has become a standard for AAM/ERP correlation analyses, the effective AAM excitation functions are first formed from six-hour AAM values in the following way:

$$
\begin{gathered}
\chi_{1}=1.43 \cdot\left(0.7 \chi_{1, p}+\chi_{1, w}\right) \\
\chi_{2}=1.43 \cdot\left(0.7 \chi_{2, p}+\chi_{2, w}\right) \\
\chi_{3}=0.7 \chi_{3, p}+\chi_{3, w}
\end{gathered}
$$

where: $\chi_{1, p} \chi_{2, p}$ are the equatorial components of the AAM excitations due to surface pressure; $\chi_{1, p} \chi_{2, p}$ are the equatorial components of the AAM excitations due to winds ( $\mathrm{up}$ to $10 \mathrm{mb} ; \chi_{3, p}$ is the axial component of the AAM excitations due to surface pressure; $\chi_{3, w}$ is the axial component of the AAM excitations due to winds (up to $10 \mathrm{mb}$ ); 
Note that two sets of values are given in (Salstein and Rosen, 1997) for surface pressure AAM components, with and without inverted barometer corrections that account for the response of oceans to changing atmospheric pressure distribution. Assuming that the AAM values refer to the middle of the six hour period, the desired $24 \mathrm{~h}$ average values were obtained by a simple integral approximation from the five values at epochs of $0,6,12,18$ and $24 \mathrm{~h}$ UT:

$$
\chi=\left(\chi_{0 h}+2.5 \chi_{6 h}+3 \chi_{12 h}+2.5 \chi_{18 h}+\chi_{24 h}\right) / 10 .
$$

Although, a simple average of the first four AAM values, gave almost identical results, likely since the adjacent six hour values are somewhat correlated, due to the underlined atmospheric models used to derived the AAM series (Salstein, 1998, priv. comm.). The above effective AAM components then can be compared to the corresponding excitation functions derived directly from the observed ERP $\left(\mathrm{PM}_{x}, \mathrm{PM}_{y}, \mathrm{PM}_{x r a t e}, \mathrm{PM}_{\text {yrate }}\right.$ and LOD) series by the following approximate expressions (Barnes et al., 1983):

$$
\begin{gathered}
\tilde{\chi}_{1}=P M_{x}+P_{\text {Chandler }} \cdot P M_{\text {yrate }} \\
\tilde{\chi}_{2}=-P M_{y}+P_{\text {Chandler }} \cdot P M_{\text {xrate }} \\
\tilde{\chi}_{3}=L O D
\end{gathered}
$$

where $P_{C h a n d l e r}$ is the assumed Chandler period ( $\sim 434$ days) . Recent studies, using the IGS ERP, have obtained correlation exceeding 0.9 for $\chi_{3}$ and about 0.6 to 0.8 for the equatorial components $\chi_{1}$ and $\chi_{2}$ (Beutler et al., 1998; Mireault et $a l ., 1999)$ ). The above equations, after dividing both sides by $P_{C h a n d l e r}$,

$$
\begin{gathered}
\tilde{\chi}_{1} / P_{\text {Chandler }}=P M_{x} / P_{\text {Chandler }}+P M_{\text {yrate }} \\
\tilde{\chi}_{2} / P_{\text {Chandler }}=-P M_{y} / P_{\text {Chandler }}+P M_{\text {xrate }}
\end{gathered}
$$

can be interpreted as the PM rates in excess to the rates of the Chandlerian motion, represented by the first term on the right hand side of the equations (9) and (10). These excess PM rates give the same PM/AAM correlation as the excitation functions, but they can also be directly compared (subject only to a constant) to the corresponding AAM excitation functions, divided by $P_{\text {Chandler }}$. A closer examination of the above excess PM rate also reveals that they only include the noise contribution of the observed PM rates and that the noise contribution due to the observed PM is reduced to a negligible level by dividing it by the Chandler period. The excess $\mathrm{PM}$ rate formulation was used throughout this contribution. Table 4 summarizes the rms of the excess PM rate and LOD differences with respect to the AAM derived PM rates for the IGS Final, IGS Rapid (IGR) and the 7 AC Final PM rate solutions during the studied period.

Both AAM values corrected (ib) and not corrected (nib) for the inverted barometer effects were used in Table 4. The ib AAM values always gave the best results for PM during this period, so that the IGS and the best PM rate compared with the AAM derived excess PM rates at about $0.3 \mathrm{mas} / \mathrm{d}$ precision level. This is remarkable since one can derive precise, daily PM rates, subject only to a constant offset, once an AAM series and precise PM solutions (which even need not to have the daily sampling since it can be smoothed and/or 
Table 4. Rms of IGS and AC ERP rate differences with respect to AAM PM rates during June 30, 1996 and December 31, 1998 with (ib) or without (nib) the inverted barometer corrections.

\begin{tabular}{lcccccc} 
AC & $\begin{array}{c}\text { Xrt(nib) } \\
\text { mas/d }\end{array}$ & $\begin{array}{c}\text { Xrt(ib) } \\
\text { mas/d }\end{array}$ & $\begin{array}{c}\text { Yrt(nib) } \\
\text { mas/d }\end{array}$ & $\begin{array}{c}\text { Yrt(ib) } \\
\text { mas/d }\end{array}$ & $\begin{array}{c}\text { LODR(nib) } \\
\mu \text { s/d }\end{array}$ & $\begin{array}{c}\text { LODR(ib) } \\
\mu \mathrm{s} / \mathrm{d}\end{array}$ \\
\hline COD & 0.486 & 0.355 & 0.627 & 0.308 & 68.7 & 71.1 \\
EMR & 0.755 & 0.684 & 0.854 & 0.648 & 76.4 & 80.6 \\
ESA & $\mathbf{0 . 7 2 2}$ & 0.586 & 0.834 & 0.555 & 75.4 & 79.0 \\
GFZ & $\mathbf{0 . 5 0 5}$ & 0.395 & 0.637 & 0.374 & 67.3 & 71.6 \\
IGR & $\mathbf{0 . 5 4 2}$ & 0.447 & 0.693 & 0.421 & 69.5 & 72.8 \\
JPL & $\mathbf{0 . 5 1 5}$ & 0.368 & 0.640 & 0.368 & 69.2 & 71.6 \\
NGS & $\mathbf{0 . 9 2 5}$ & 0.866 & 1.232 & 0.961 & 100.3 & 106.9 \\
SIO & $\mathbf{0 . 4 9 4}$ & 0.347 & 0.628 & 0.310 & 69.1 & 71.9 \\
IGS & $\mathbf{0 . 4 9 2}$ & 0.353 & 0.631 & 0.314 & 66.6 & 69.8 \\
\hline
\end{tabular}

interpolated at a mas level). LOD comparisons show about the same agreement for both ib and nib values, though all the LODR differences in Table 4 had to be corrected for a common rate and semiannual signal. During this relatively short period, an apparent LODR drift and semiannual signal with respect to AAM could be caused by unmodelled oceanic or core-mantle coupling processes and the missing AAM contribution of stratospheric winds above the $10 \mathrm{mb}$ layer, respectively (Rosen et al., 1990; Dickey et al., 1994).

Figures 4 and 5 show spectra of the PM rate differences with respect to the AAM derived ERP rates for IGS, IGR and ACs, during the 17-month period of August 1997 to December 1998. The PM x rate spectra are quite well behaved, in particular for the more precise group of ACs shown in the bottom part of Figure 4. (note the difference in the scale between the top and bottom parts). The exceptions are several pronounced peaks for GFZ AC in the 2 to 4 day period bands and a large, 14.2-day peak for JPL AC. None of these periods are seen in the PM x spectra in Figure 2, which may indicate that they are of a mismodeling origin. (Note a PM rate amplitude of a 14 day period would have to be $14 /(2 \pi)=2.2$ times larger in the PM x spectra of Figure 2 (e.g. Rothacher et al., 1999)). The anomalistic behavior of JPL PM solutions in Figures 4 and 5 may be caused by the use of an older sub daily PM model (Herring and Dong, 1994; Zumberge, 1999, priv. com.) instead of the IERS standard one (McCarthy, 1996; Ray et al., 1994) used by most ACs. Nevertheless the agreement amongst $\mathrm{ACs}$ for periods between 20 and 100 days in the bottom group of $\mathrm{ACs}$ is quite remarkable and may indicate a real signal of a non-AAM origin, or common mode errors.

The PM y spectra in Figure 5 show similar behavior for all ACs, including the same 14.2-day peak for JPL and several, high frequency period peaks for GFZ. Additionally, a small annual signal is indicated for IGS and most ACs here. An interesting feature in Figure 5 is the 11.9-day peak with amplitude of about $0.08 \mathrm{mas} / \mathrm{d}$ seen for most $\mathrm{AC}$ solutions. 

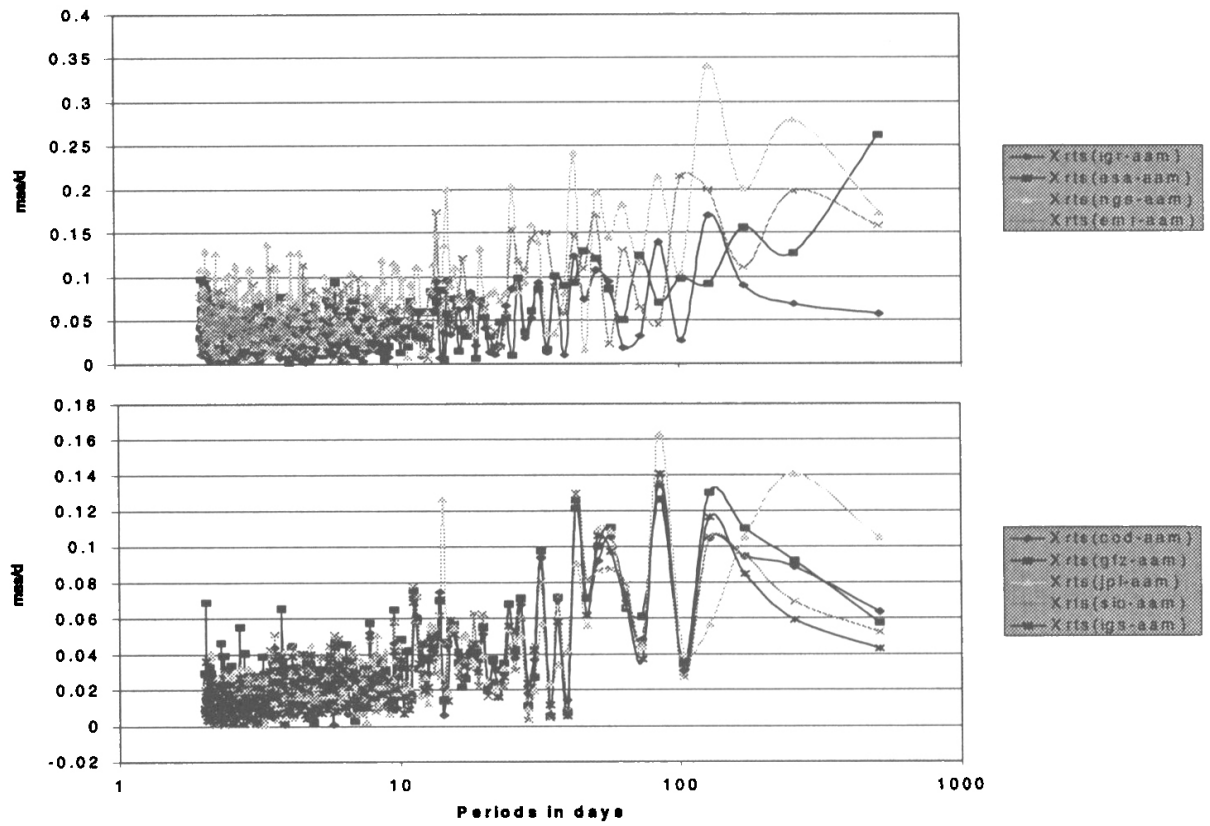

Figure 4. AC PM x rate amplitude and periods with respect to Atmospheric Angular Momentum (AAM) derived PM x rates. 


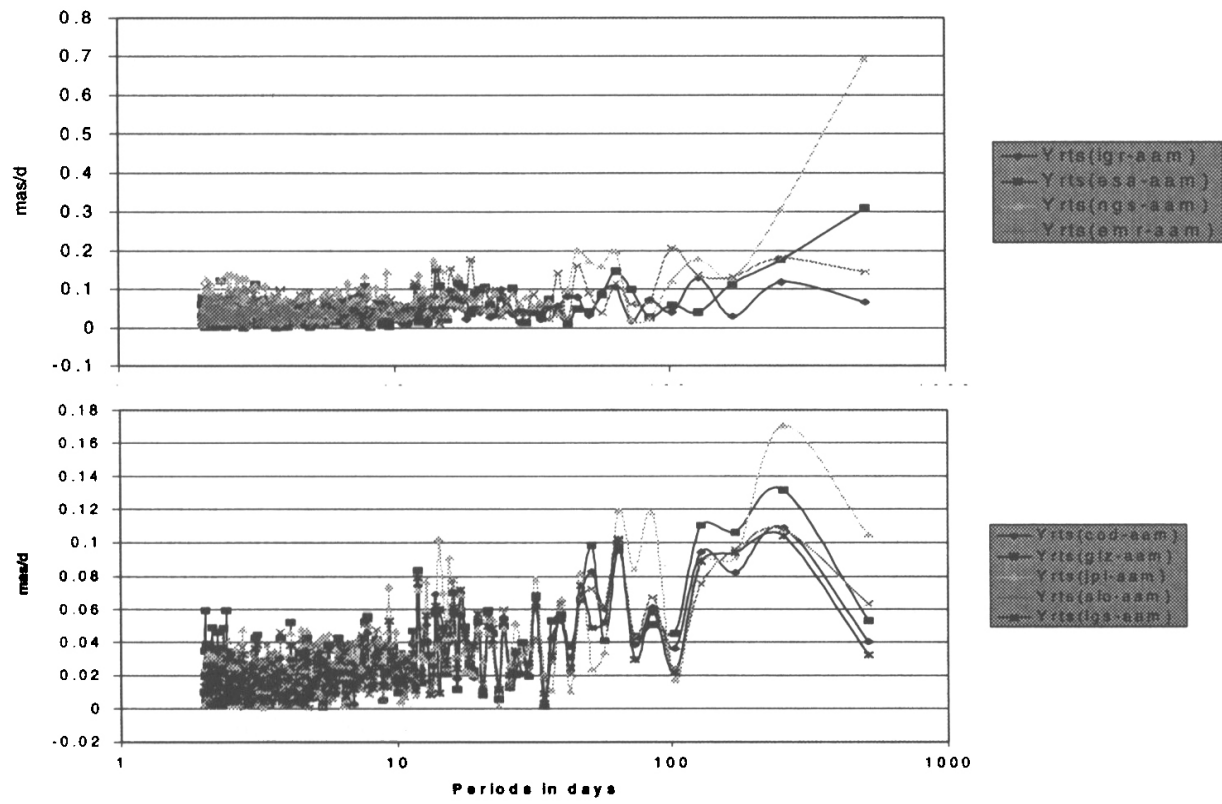

Figure 5. AC PM y rate amplitude and periods with respect to Atmospheric Angular Momentum (AAM) derived PM y rates. 
Table 5 shows annual means for IGS and AC ERP correlation with AAM during 1998, both with (ib) and with no inverted barometer (nib) corrections. As seen in the Table 5, IGS and the best ACs show the highest correlation with the ib AAM, namely better than $0.8,0.6$ and 0.9 for PM x, y rates and LODR, respectively.

Table 5. Correlation of AC Earth Rotation Parameter (ERP) rate solutions and AAM with (ib) and without (nib) inverted barometer corrections during 1998.

\begin{tabular}{lcccccc} 
AC & $\begin{array}{c}\mathrm{Xrt} / \chi_{2} \\
\text { nib }\end{array}$ & $\begin{array}{c}\mathrm{Xrt} / \chi_{2} \\
\mathrm{ib}\end{array}$ & $\begin{array}{c}\text { Yrt } / \text { chi } \\
\text { nib }\end{array}$ & $\begin{array}{c}\text { Yrt } / \chi_{1} \\
\mathrm{ib}\end{array}$ & $\begin{array}{c}\text { LODR } / \chi_{3} \\
\text { nib }\end{array}$ & $\begin{array}{c}\text { LODR/ } / \chi_{3} \\
\text { ib }\end{array}$ \\
\hline COD & 0.792 & 0.836 & 0.358 & 0.601 & 0.953 & 0.955 \\
EMR & 0.597 & 0.631 & 0.206 & 0.378 & 0.952 & 0.954 \\
ESA & 0.512 & 0.566 & 0.144 & 0.436 & 0.920 & 0.923 \\
GFZ & 0.776 & 0.817 & 0.372 & 0.536 & 0.956 & 0.958 \\
IGR & 0.741 & 0.759 & 0.276 & 0.475 & 0.955 & 0.956 \\
JPL & 0.762 & 0.805 & 0.346 & 0.484 & 0.952 & 0.954 \\
NGS & 0.500 & 0.525 & -0.035 & 0.346 & 0.947 & 0.948 \\
SIO & 0.784 & 0.835 & 0.356 & 0.599 & 0.955 & 0.956 \\
IGS & 0.786 & 0.834 & 0.353 & 0.601 & 0.954 & 0.956 \\
\hline
\end{tabular}

The correlation of ERP with AAM varies considerably, depending on the time of the year, the averaging intervals and the variability of the excess ERP rate signal. Figure 6 demonstrates the point by showing the correlation, averaged over 30-day intervals, as a function of time and the standard deviations of the ERP rates (SigXrt, SigYrt and SigLOD, i.e. the square root of the denominators of the correlation function). As expected, the IGS combined LOD, which is only available from March, 1997, shows the highest correlation, though, during intervals with small SigLOD (see the scale on the right hand side of Figure 6), the LODR/AAM correlation falls close to, or even below 0 . Similarly, the PM rate correlation is inversely proportional to the corresponding $\mathrm{PM}$ rate variations (standard deviations). It is interesting to note that both PM components, including the $\mathrm{PM}$ y rate $/ \chi_{1}$ pair show high correlation, at times exceeding 0.8 . Note that the variation of PM/AAM correlation in the period range of 20-150 days has been attributed to dominant ocean temperature anomalies in the El Niño regions of the tropical Pacific ocean (Kolaczek, et al., 1999). Since the ib AAM consistently gave the best correlation results (see Table 5), only the ib AAM values were used in Figure 6 and the subsequent figures. This should be expected as for the periods longer than 10 days the inverted barometer assumption should hold (Ponte et al., 1997)

The spectra of the ERP rate signal remaining after the removal of the AAM contribution are shown in Figure 7. The amplitudes below 10 days are quite small and well behaved. PM x rates differences show three, progressively increasing peaks at about 32,43 and 85 day periods.

The PM y rate, apart from the anomalistic PM y rate signals with the annual, semiannual and 64 day periods, also has high amplitude at about 12 


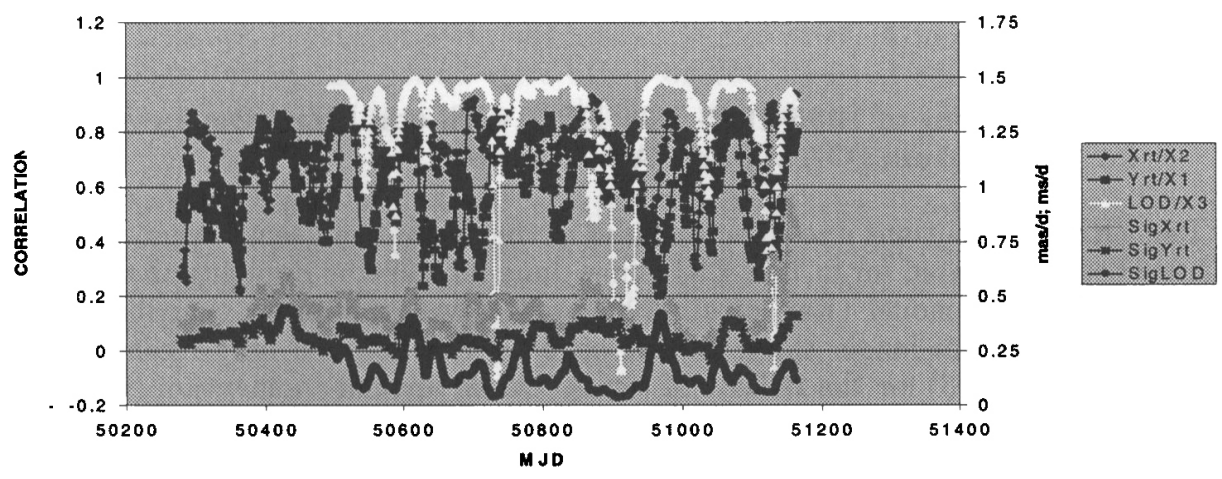

Figure 6. 30-day correlation of IGS Final ERP rates and AAM (inverted barometer) during June 30, 1996 to December 31, 1998. Also shown are 30-day LODR and excess PM rate variations (sigmasSigLOD, SigXrt, SigYrt).
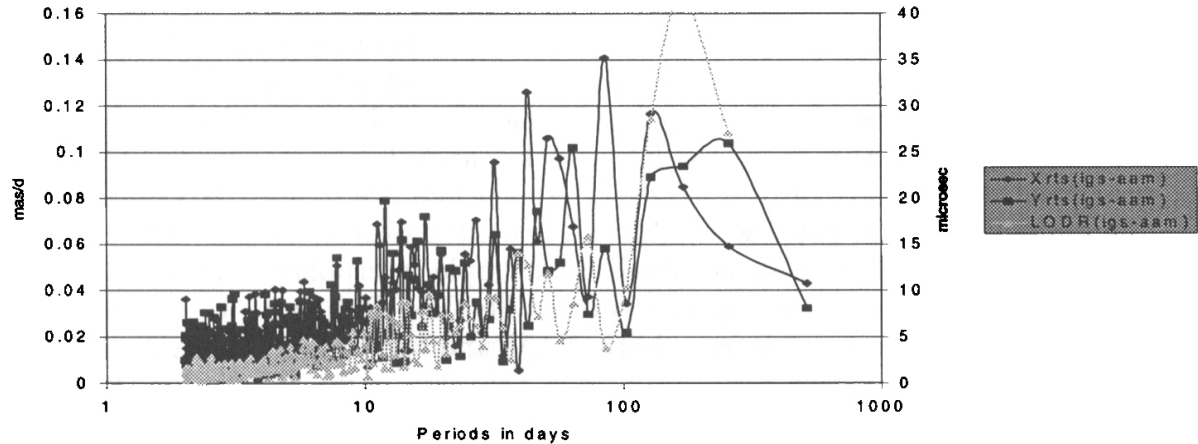

Figure 7. Amplitudes and periods of IGS Final ERP rate differences with respect to inverted barometer AAM derived ERP rates during 1998. 
day-period. The LODR spectrum is better behaved, with the exception of the pronounced semiannual period, indicating that the removed empirical signal with the semiannual period was inadequate. The remaining ERP rate signal shown in Figure 7 may be, to the large extent, due to neglected ocean angular momentum (OAM). Some global geophysical phenomena such as solar winds, the largest earthquakes (e.g. Clark et al., 1998), or simply systematic errors present in the IGS ERP and/or AAM series may have also contributed to signals seen in Figure 7.

\section{Comparisons of 1995-1996 IGS ERP with combined Ocean Angu- lar Momentum (OAM) and AAM series.}

The ERP contributions due to ocean tides in the diurnal and semidiurnal bands have been accounted for in IGS ERP since June 30,1996 by applying the IERS sub- daily ERP model (Ray et al., 1994). However, the ocean contributions to ERP at periods longer than 10 days have been established only recently by Ponte et al. (1998) for the IERS PM series. Ocean angular momentum (OAM) series, unlike those of AAM, are not generally available, since they require considerable computation and modeling effort. The OAM series, compatible with the formulation of Barnes et al. (1983) and used in Ponte et al. (1998), was kindly made available for this investigation by Ponte (1999, priv. com.).

The OAM series is based on a recent numerical ocean model (Marshall et al., 1997), driven once and twice daily by surface heat, fresh water fluxes and wind stress fields from National Center for Environment Prediction (NCEP). However, no atmospheric pressure data was included in this model. Thus, the OAM series is consistent with the application of the inverted barometer model corrections for periods longer than 10 days (Ponte et al., 1998). The OAM series with a 5 day sampling covers the period of 1985 to April, 1996 , so that only the period common with the IGS combined PM series, covering January 1995 to April 1996, was used for this OAM and AAM comparison studies.

During this period the IGS combined daily ERP did not include any ERP rate (i.e. PM rate and LOD) solutions and did not yet account for the sub-daily ERP model. Since the IGS PM daily series is based on a combination of the $7 \mathrm{AC}$ solutions averaged over $24 \mathrm{~h}$, neglecting of the sub-daily PM model is not expected to have any significant effect on the IGS PM and the derived PM rates. The desired IGS PM rates at the PM epochs (noon UT) were simply obtained as a half of the difference between the subsequent and preceding PM values, so that the derived IGS PM rates were, in fact, averaged over 48 rather than 24 hours. The daily OAM values were obtained by a simple interpolation from the original OAM series with 5-day sampling interval. The IGS PM rates were compared to the PM rates derived rates from both AAM and the combined OAM and AAM (O+AAM) series, in the same way as in the previous section. The 1995-1996 comparisons are summarized in Table 6 which shows means and rms (standard deviations around the means) for both AAM series, i.e. both with (ib) and without (nib) inverted barometer corrections. Similarly as seen in Table 4 for the 1996-1998 period, the comparisons based on the ib AAM values gave the best results. Furthermore, the addition of the OAM significantly improved rms for both PM x and y rates. 
The corresponding correlation for both AAM and O+AAM with the IGS ERP rates is listed in Table 7. Unlike the 1996-1998 period (Table 5) and Table 6 where the ib AAM gave the best results, the nib AAM shows slightly higher correlation for all ERP rate components than the ib AAM. However, when OAM is accounted for, then once again the ib $O+A A M$ gave the highest correlation, almost 0.8 for both PM rate components. Since OAM series contains only the equatorial components, there are no O+AAM LOD correlation values listed in Table 7. Note that the IGS LOD were also obtained by differencing the adjacent IGS UT values, in the same way as for the IGS PM rates. Also note that the IGS UT values during this period were based on the observed and/or interpolated Bulletin A UT1 series that were available at the time of IGS PM combinations.

Table 6. IGS PM $\mathrm{x}$ and $\mathrm{y}$ rate differences with respect to AAM and the combined OAM and AAM (O+AAM) based on no inverted barometer (nib) and the inverted barometer (ib) correction model during January 1995 and April 1996.

\begin{tabular}{|c|c|c|c|c|c|c|c|c|}
\hline & \multicolumn{2}{|c|}{ Xrt(nib) } & \multicolumn{2}{|c|}{$\mathrm{Xrt}(\mathrm{ib})$} & \multicolumn{2}{|c|}{ Yrt(nib) } & \multicolumn{2}{|c|}{ Yrt(ib) } \\
\hline & $\begin{array}{l}\text { Mean } \\
\text { mas } / d\end{array}$ & $\begin{array}{c}\mathrm{rms} \\
\mathrm{mas} / \mathrm{d}\end{array}$ & $\begin{array}{l}\text { Mean } \\
\text { mas } / d\end{array}$ & $\begin{array}{c}\mathrm{rms} \\
\mathrm{mas} / \mathrm{d}\end{array}$ & $\begin{array}{l}\text { Mean } \\
\text { mas } / d\end{array}$ & $\begin{array}{c}\mathrm{rms} \\
\mathrm{mas} / \mathrm{d}\end{array}$ & $\begin{array}{l}\text { Mean } \\
\text { mas/d }\end{array}$ & $\begin{array}{c}\mathrm{rms} \\
\mathrm{mas} / \mathrm{d}\end{array}$ \\
\hline AAM & -0.040 & 0.583 & -0.018 & 0.464 & 0.019 & 0.553 & -0.020 & 0.312 \\
\hline O+AAM & -0.064 & 0.508 & -0.042 & 0.332 & -0.023 & 0.556 & -0.061 & 0.232 \\
\hline
\end{tabular}

Table 7. Correlation of IGS ERP rate with AAM and the combined OAM and AAM (O+AAM) based on no inverted barometer (nib) and the inverted barometer (ib) correction model during January 1995 and April 1996.

\begin{tabular}{lcccccc} 
& $\begin{array}{c}\mathrm{Xrt} / \chi_{2} \\
\text { nib }\end{array}$ & $\begin{array}{c}\mathrm{Xrt} / \chi_{2} \\
\mathrm{ib}\end{array}$ & $\begin{array}{c}\text { Yrt } / \chi_{1} \\
\text { nib }\end{array}$ & $\begin{array}{c}\text { Yrt } / \chi_{1} \\
\text { ib }\end{array}$ & $\begin{array}{c}\text { LOD } / \chi_{3} \\
\text { nib }\end{array}$ & $\begin{array}{c}\text { LOD } / \chi_{3} \\
\mathrm{ib}\end{array}$ \\
\hline AAM & 0.655 & 0.617 & 0.601 & 0.577 & 0.924 & 0.920 \\
O+AAM & 0.729 & 0.789 & 0.684 & 0.785 & & \\
\hline
\end{tabular}

As already noted earlier, the PM correlation varies considerably with time and signal variation. This is again demonstrated in Figures 8 and 9 that show 30-day correlation of PM $x$ and PM $y$ rates with both the AAM and O+AAM series. As in Figure 6, the corresponding signals magnitudes (the square root of the correlation denominator) are also shown in Figures 8 and 9. As expected, accounting for OAM also significantly improves the 30-day correlation. While the highest correlation often exceeds 0.9 for both PM x and y rates, the low correlation is almost always accompanied by low magnitude signals. However even for such low signal variations the addition of OAM almost always, sometimes dramatically, improves the correlation.

Table 8 lists the correlation of the PM/AAM and PM/O+AAM as a function of the intervals. It is remarkable that both AAM and O+AAM show high correlation of about 0.6 , even during short intervals of only 6 days. Figure 10 
Table 8. Correlation of IGS combined PM rate with AAM and the combined OAM and AAM (O+AAM) (inverted barometer) for different period bands during January 1995 and May 1996.

\begin{tabular}{lcccc} 
& \multicolumn{2}{c}{ AAM } & \multicolumn{2}{c}{ O+ AAM } \\
periods & Xrt $/ \chi_{2}$ & Yrt $/ \chi_{1}$ & Xrt $/ \chi_{2}$ & Yrt $/ \chi_{1}$ \\
\hline ALL & 0.617 & 0.577 & 0.789 & 0.785 \\
$<60 \mathrm{~d}$ & 0.700 & 0.592 & 0.801 & 0.769 \\
$<30 \mathrm{~d}$ & 0.664 & 0.611 & 0.769 & 0.751 \\
$<10 \mathrm{~d}$ & 0.650 & 0.571 & 0.717 & 0.636 \\
$<6 \mathrm{~d}$ & 0.617 & 0.519 & 0.667 & 0.570 \\
\hline
\end{tabular}

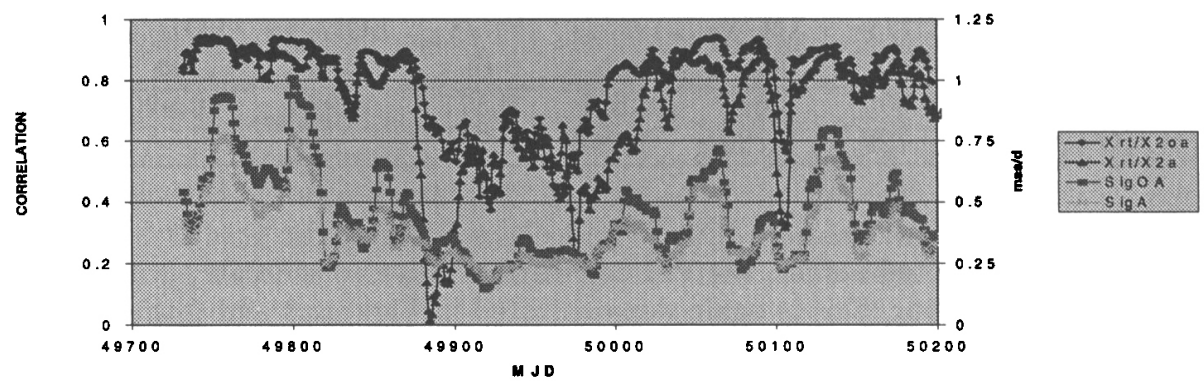

Figure 8. 30-day correlation of IGS combined $\mathrm{PM} \times$ rates with $O+\operatorname{AAM}\left(\mathrm{Xrt}_{\mathrm{r}} / \chi_{1}\right.$ oa) and AAM (Xrt $/ \chi_{1}$ a) for January 1995 to April 1996. Also shown are 30-day variations (sigmas) corresponding to O+AAM (SigOA) and AAM (SigA). 


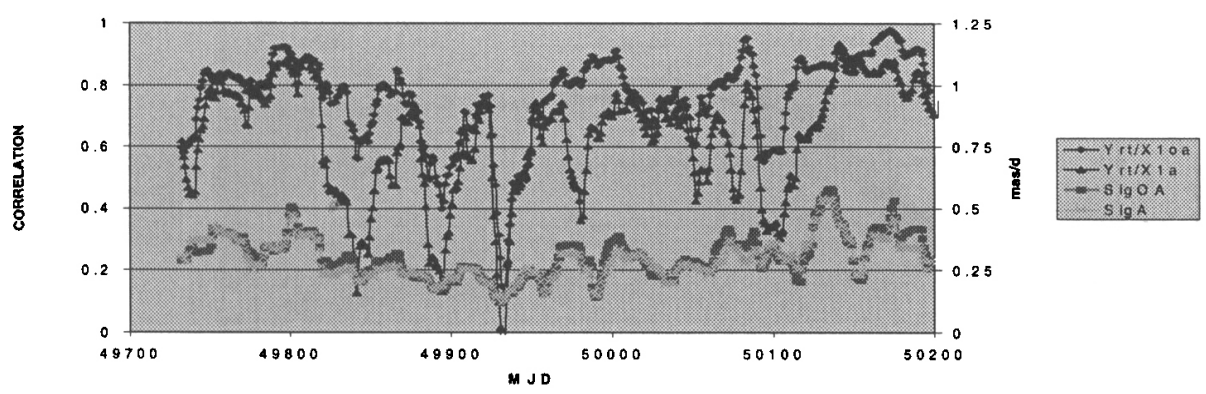

Figure 9. 30 day correlation of IGS combined PM y rates with OAM+AAM (Yrt/ oa) and AAM (Yrt/ a) for January 1995 to April 1996. Also shown are 30-day variations (sigmas) corresponding to O+AAM (SigOA) and AAM (SigA).

shows a cumulative correlation of $\mathrm{PM} / \mathrm{O}+\mathrm{AAM}$ as a function of retained periods. As in Table 7, significant correlation are seen in Figure 10, already when periods up to six days are retained and at about 14 day period a correlation of about 0.8 is reached for all the PM components.

Figures 11 and 12 show phase coherence (cosine of the phase lags) and amplitude comparisons for IGS PM rates, AAM and O+AAM, during the period of January 1995 to April 1996. In an ideal case, the phase lag should be equal to zero (the coherence equal to 1) and the corresponding amplitudes should be the same. Phase coherence improves significantly for periods above 6 days once OAM is accounted for, though there are some notable exceptions. For the PM x rate, only the low coherence periods of about 10.4 and 36.6 days are meaningful since the corresponding amplitudes are not negligible. Another interesting feature is the new coherence low at about 85 days for O+AAM despite the small increase in the corresponding O+AAM amplitude. A similar, 85-day anomaly, though on a smaller scale, is also seen for PM y in Figure 12. Other low coherence periods for PM y include 13.5, 16 and 51 days, in all cases only IGS PM y rate amplitudes are significant. Since there is no corresponding signal seen in neither AAM nor O+AAM, it may be caused by systematic errors in PM $\mathrm{y}$ solutions. No comparable signal is seen for the PM x solutions, which tend to be stronger due to the geometry of the IGS station network. This is further supported by an apparent annual term in PM y after the O+AAM contribution is removed. The IGS PM reference datum during this period was based on the ITRF93 position and velocities of the same 13 ITRF stations. Small errors in several, less precise ITRF93 station positions and velocities could cause a small annual signal in IGS PM since GPS satellite geometry also have an annual cycle. However, the semiannual terms seen for AAM in both PM x and PM y are greatly diminished by the addition of OAM, which is quite encouraging. 


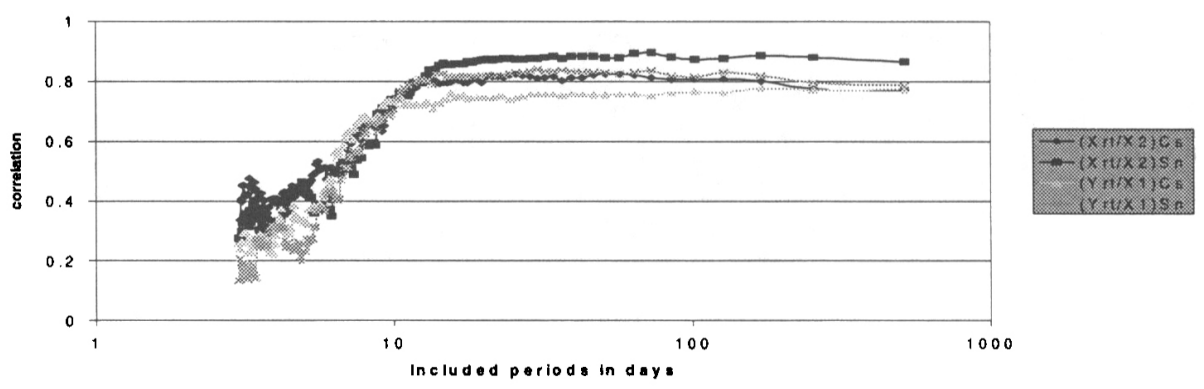

Figure 10. Cumulative correlation of OAM + AAM (inverted barometer) and IGS PM rate cosine (Cs) and sine (Sn) spectral components (January 1995 to April 1996).

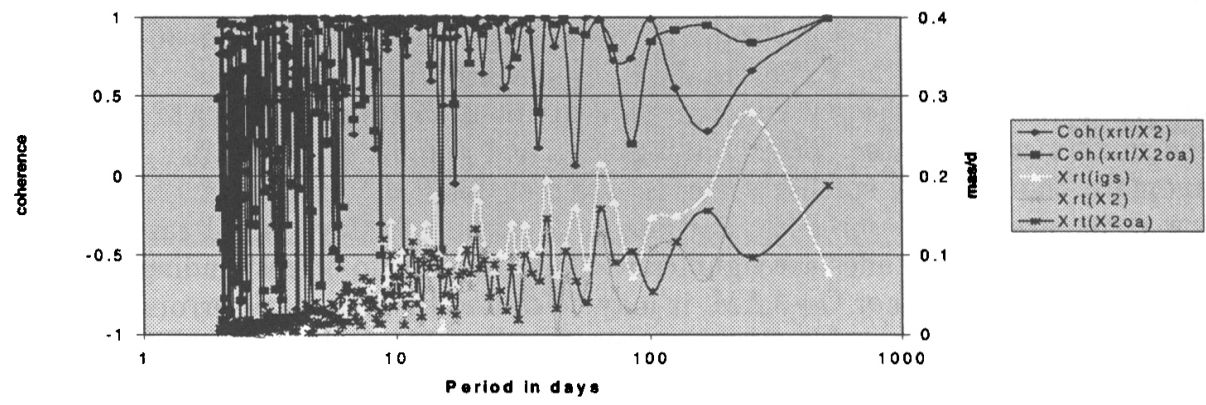

Figure 11. IGS PM x rate, AAM $\left(\chi_{2}\right)$ and OAM + AAM $\left(\chi_{2}\right.$ oa $)$ amplitudes and phase coherence (cosine of phase lag) during January 1995 to April 1996. 


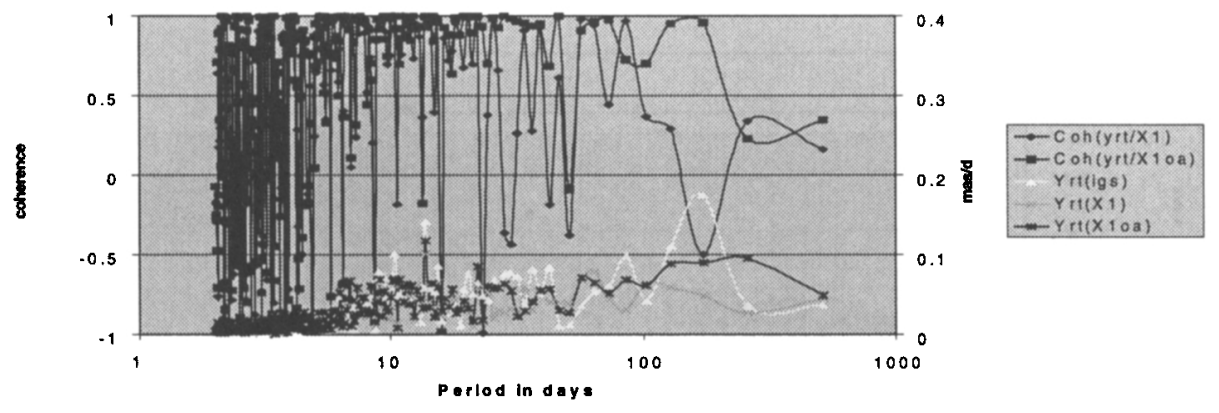

Figure 12. IGS PM y rate, AAM $\left(\chi_{1}\right)$ and OAM +AAM $\left(\chi_{1}\right.$ oa $)$ amplitudes and phase coherence (cosine of phase lag) during Jan 1995 to April 1996.

Figure 13 shows the power spectra of IGS PM rate differences with respect to O+AAM. It is interesting to note that the geometrically stronger PM x solutions show significantly higher amplitudes than PM y solutions for periods above 10 days, indicating that the remaining signal unaccounted for by O+AAM in $\mathrm{PM} \mathrm{x}$ rate may be real. Also interesting to note is the apparent and atypical amplitude decrease for periods just above the 10-day period. This may be indicative of the residual ocean contributions at or below 10 day periods, which could not be removed due to the 5-day sampling (smoothing) of the OAM series used. A 5-day smoothing almost completely suppresses signals with periods at or below 5 days, and even the amplitudes of the 10 day period are reduced down to the $60 \%$ level (e.g. Rothacher et al., 1999).

The 14-day signal seen for both PM x and PM y may be due to IGS PM solution problems (e.g. the neglect of ocean loading, inadequate solid Earth tide modeling etc.). The remaining apparent PM x signals at 21.3, 36.6, 51.2 and 85 -day periods may or may not be real and may indicate effects other than of atmospheric or oceanic origins. From these PM x peak periods, only the 36.6 and 85-day periods have the corresponding peaks in the PM y spectrum. For completeness, note that the PM amplitudes can readily be computed by multiplying the rate amplitude with the following ratio: period $(\mathrm{d}) /(2 \pi)$ (Rothacher et al., 1999). For example, the significant peak of $.103 \mathrm{mas} / \mathrm{d}$ at the 36.6 day period corresponds to a PM x amplitude of .600 mas and PM x rate amplitude of .115 at 85.3 day period gives an amplitude of 1.561 mas in PM x. The annual signals seen in both PM x and PM y rates that cannot be due to solution artifacts are somewhat puzzling, since the corresponding PM amplitudes would have to be about 60 times larger, i.e. about 10 mas. The seasonal amplitudes observed in IGS PM are well below the 1 mas level (IERS Annual Reports for 1995, 1996). Another indication that this annual amplitude may be due to errors in AAM (e.g. pressure corrupted by seasonal water changes (e.g. Rosen and Salstein, 


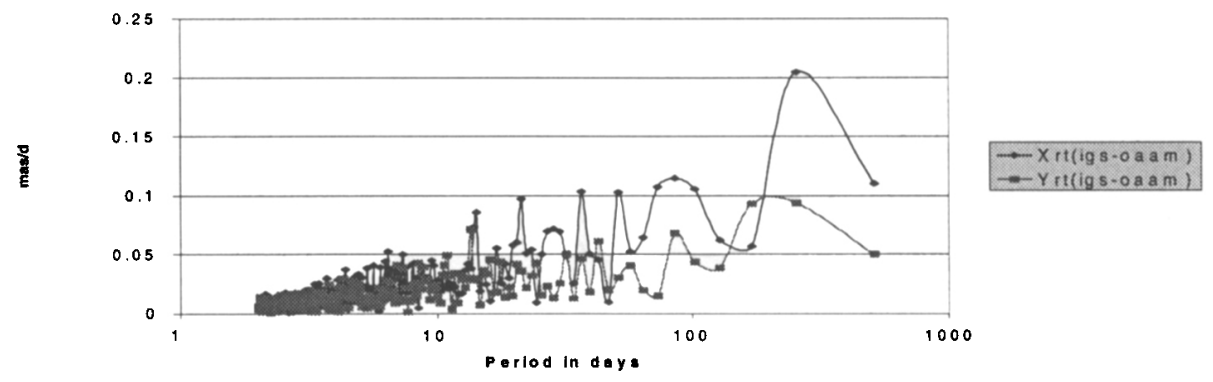

Figure 13. Amplitudes and periods of IGS Polar Motion rate and AAM + OAM derived PM rate differences during January 1995 to April 1996.

1991)), or the seasonal effects in OAM, is the fact that the addition of OAM increases the seasonal amplitudes of residual (IGS - (O+AAM)). This can be seen from the corresponding (IGS, AAM and O+AAM) seasonal amplitudes in Figures 11 and 12 .

\section{Other geophysical contributions to PM}

Additional possible contributions to the $\mathrm{PM}$ rate signal could be due to solar wind (Barnes et al., 1983) that is related to ionospheric variations, which in turn may induce atmospheric changes, as well as the contributions of large earthquakes (e.g. Chao et al., 1995). At least two (i.e. 36.6, 51.2) and possible the 85-day period peaks discussed earlier can also be seen in the spectrum for the independent daily series of the $\mathrm{C}_{22}$ coefficients of the spherical expansions of TEC (total electron content) that are made available by CODE AC (Schaer, 1999). In fact the agreement between $C_{22}$ and $P M \times$ rate residual amplitudes in the 30 to 60 day band is quite remarkable, as seen from Figure 14 which shows spectra of $P M$ rate residuals with respect to $0+A A M$ and $\mathrm{C}_{22}$. Note that the PM x rate peak at the 21.3-day period is also clearly seen in the $\mathrm{C}_{20}$ spectrum (not shown here). The above PM x rate peaks lag in phase by about 2 weeks. Analogously to the Earth gravity field spherical harmonic expansions, the $\mathrm{C}_{20}$ variations can be viewed as shape variations of the TEC body, while the $\mathrm{C}_{22}$ is related to the longitudinal rotation of the TEC body. Figure 14 also shows large amplitude peaks at a 14 -day period for both $\mathrm{PM}$ rate components and $\mathrm{C}_{22}$, indicating that it may be of the same origin (the Moon).

The response of the Earth rotational dynamics to earthquake induced impulses varies considerably, depending on geographical locations as well as earthquake characteristics, such as magnitude, focal mechanism, total energy released, etc. Resulting PM changes are accumulated for up to ten days and PM changes 

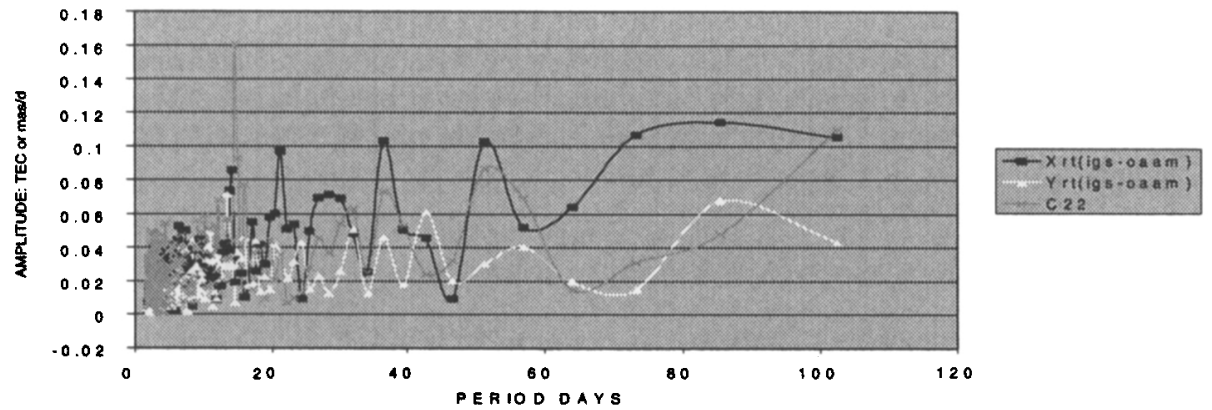

Figure 14. Spectra of IGS PM Rate residuals with respect to $\mathrm{O}+\mathrm{AAM}$ and the daily $\mathrm{C}_{22}$ (TECU) spherical harmonic coefficient series of TEC produced by CODE AC.

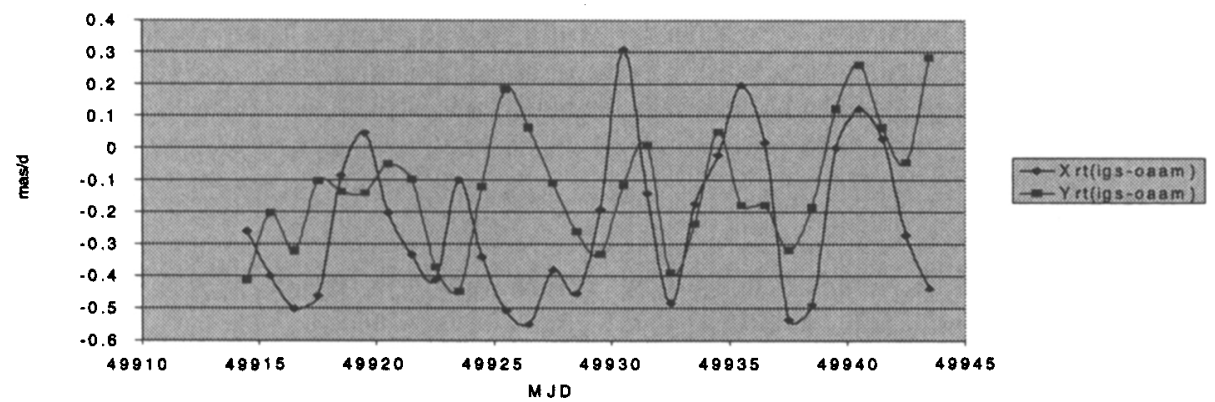

Figure 15. IGS and O+AAM residuals 15 days before and after the Chile Earthquake (MJD 49929, magnitude 8.0, depth $45 \mathrm{~km}$ ). 
can even precede large earthquakes by a few days (Chao et al., 1994). Of the ten largest world earthquakes of the decade, all magnitude 8 or greater, listed at wwweic.cr.usgs.gov/neis/eqlists/last_big10.html, four are in the period for which both IGS PM and OAM were available (MJD 49815, 49929, 50000 and 50130). Figure 15 shows the corresponding 30-day portion (centered at the event) of the IGS PM rate - (O+AAM) residuals, for one of the above earthquakes with the greatest depth i.e. the Chilean Earthquake of July 30, 1995 (MJD 49929). It clearly shows the IGS PM rate residuals for the deepest earthquake, the Chilean Earthquake of July 30,1995 (MJD 49929). Clearly it shows that both PM $\mathrm{x}$ and $\mathrm{y}$ components are disturbed with an almost identical, highly periodical signal. However, the remaining three great earthquakes of the studied interval do not seem to imply any such excitation of PM rates with periodical signals.

\section{Summary}

The IGS combined PM and PM rates, due to high precision and daily resolution, are ideally suited for AAM and OAM studies and comparisons. During January 1995- April 1996 when both OAM and AAM were available and could be accounted for, a high degree of correlation of about 0.8 for both PM $\mathrm{x}$ and y components was observed. The high correlation persisted even for periods as short as six days. The residual PM rate signal with respect to OAM+AAM had rms of about 0.2 to $0.3 \mathrm{mas} / \mathrm{d}$ with some periodical signal with pronounced amplitudes at the fortnight to seasonal period bands. All significant amplitude peaks of the $\mathrm{O}+\mathrm{AAM} / \mathrm{PM}$ rate residuals, except for the seasonal ones, had the corresponding amplitude peaks also in the ionospheric TEC variations and may, in fact, be indicating a real signal of the ionosheric origin. The effect of the largest earthquakes is difficult to ascertain, perhaps with the exception of the deepest $(45 \mathrm{~km}$ ), Chilean earthquake of July 1995 which showed a regular periodical signal in both $\mathrm{PM}$ components which persisted for about 15 days after the earthquake.

Acknowledgments. Most of the research was done at the Astronomical Institute of University of Bern in early 1999, while the first author was a visiting scientist at the institute. The authors would like to express their thanks to Dr. R.M. Ponte of Atmospheric and Environmental Research, Inc. of Cambridge, Mass., for making the OAM series available to us.

\section{References}

Barnes, R.T.H., R. Hide, F.R.S., A.A. White and C.A. Wilson, 1983, Proc. R. Soc. Lond., Ser. A, 387, pp. 31-73.

Beutler, G., M. Rothacher, T. Springer, J. Kouba and R.E. Neilan, 1998, 32nd COSPAR Scientific Assembly, Nagoya, Japan, July12, in Advances in Space Research (in press).

Beutler, G, 1997, Rotation der Erde: Theorie, Methoden, Resultate aus Satellitengeodsie und Astrometrie, Astronomisches Institut der Universitt Bern.

Beutler, G., J. Kouba and T. Springer, 1995, Bull. Geod. 69, pp. 200-222. 
Boucher, C, Z. Altamimi and P. Silliard, 1998, IERS Technical Note No. 24, Central Bureau of IERS, Observatory of Paris, France, May.

Chao, F.B. and R.S. Gross, 1995, Geophys. J. Int., 122, 776-783.

Clark, T.A., C. Ma, J.W. Ryan, B.F. Chao, J.M. Gipson, D.S. MacMillan, N.R. Vandenberg, T.M. Eubanks, and A.E. Neill, 1998, EOS, 79, April 28, $205 / 209$.

Dickey, J.O., S.L. Marcus, R. Hide, T.M. Eubanks and D.H. Boggs, 1994, J. Geophys. Res., 99, pp 23921-23937.

Eubanks, T.M. J.A. Steppe and J.O. Dickey, 1988, The Earth's Rotational and Reference Frames for Geodesy and Geodynamics, pp. 365-371.

Herring, T.A. and D. Dong, 1994, J. Geophys. Res., 99, pp 18051-18071.

International Earth Rotation Service, 1996, 1995 IERS Annual Report, Observatoire de Paris.

International Earth Rotation Service, 1997, 1996 IERS Annual Report, Observatoire de Paris.

Kolaczek, B., M. Nuzhdina, J. Nastula and W. Kosek, 1999, IERS Technical Note 26, Observatoire de Paris.

Kouba, J., 1999. IGS 1998 Annual Report, pp.13-17.

Kouba, J. 1996, 1996 IGS Analysis Center Workshop Proceedings, Silver Spring, MD, USA, March 19-21, pp. 33-42.

Kouba, J. and Y. Mireault, 1996, IGS 1995 Annual Report, pp. 45-76.

Kouba, J. and Y. Mireault, 1997, IGS 1996 Annual Report, pp. 55-100.

Kouba, J., Y. Mireault, G. Beutler, T. Springer and G. Gendt, 1998, GPS Solutions, 2, pp. 3-15.

Langely, R.B., R.E. King, I.I. Shapiro, R.D. Rosen and D.A. Salstein, 1981, Nature, Lond. 294, pp. 730-732.

Marshall, J., C. Hill, L. Perelman, and C.A. Hesley, 1997, J. Geophys. Res., 102, 5733-5766.

McCarthy, D.D., 1996, IERS Technical Note 21. Observatoire de Paris.

Mireault, Y., J. Kouba and J.R. Ray, 1997, EOS Trans. $A G U, \mathbf{7 8 ( 1 7 ) , ~ S p r i n g ~}$ Meeting Suppl., S109.

Mireault, Y., J. Kouba and J.R. Ray. 1999, GPS Solutions (in press).

Ponte, R.M. 1997, Geophys., J. Int., I30, 469-474.

Ponte, R.M., D. Stammer, J. Marshall, 1998, Nature, 391, Jan. 29, pp. 476-479.

Ray, R.D., Steinberg, B.F.Chao and D.E. Cartwright, 1994, Science, 264, pp. 830-832.

Rosen, R.D. and D.A. Salstein, 1985, J. Geoghys. Res., 90, 8033-8041.

Rosen, R.D. and D.A. Salstein, 1990, J. Geoghys. Res., 95, 265-279.

Rosen, R.D. and D.A Salstein, 1991, Geoghys. Res. Lett., 18, 1925-1926.

Rothacher, M., G. Beutler, T.A. Herring, R. Weber, 1999, J. Geophys. Res. (in press).

Salstein, D.A. and R.D. Rosen, 1997, 7th Conf. On Climate Variations, American Meteorological Society, Boston, MA, pp. 344-348. 
Salstein, D.A., D.M. Kann, A.J. Miller and R.D. Rosen, 1993, Bulletin American Meteorological Society, 71, pp. 67-80.

Schaer, S., 1999, PhD Thesis, Astr. Institute of Univ. Berne, Switzerland, March.

Springer, T.A. and G. Beutler, 1993, 1993 IGS Analysis Center Workshop Proceedings, Astr. Institute University of Berne, March 25-26, pp. 242-249. 